\title{
FUZZY ZAGREB INDICES AND SOME BOUNDS FOR FUZZY ZAGREB ENERGY
}

\author{
MAHESH KALE*, S. MINIRANI \\ Department of Basic Sciences and Humanities, MPSTME, SVKM's NMIMS Deemed to be University, \\ Mumbai, India
}

*Corresponding author: mnk.maths@gmail.com

\begin{abstract}
Topological indices $M_{1} / M_{2}$ known as first/second Zagreb indices are defined as the sum of the sum/product of degrees of pairs of adjacent vertices of a simple graph. These indices and their properties have been studied in detail under chemical graph theory. In this paper we introduce the concepts of first, second and hyper Zagreb indices of fuzzy graphs. We also study the Zagreb matrices and the associated Zagreb energies of fuzzy graphs. Some bounds for these energies are also obtained.
\end{abstract}

\section{INTRODUCTION}

Topological indices are numerical quantities of structural molecular graphs. They are studied and applied in various fields by engineers, pharmacist, graph theorist and mathematicians. I. Gutman [1] in 1972, introduced the first Zagreb index and Randec in [2] introduced Randec index, which are oldest among the topological indices. I Gutman, Eliasi, Kulli, KC Das and many other experts have contributed in the developments of different Zagreb indices, Randic indices of simple graphs.

In case of classical graphs, both the vertices and edges have membership value one, but in case of fuzzy graphs both vertices and edges are equally important along with their fuzzy membership values. If the description of objects or their relationships or both are vague in nature, then we design a Fuzzy Graph model. In 1965, Zadeh [3] introduced the concept of fuzzy sets and fuzzy relations. Further Rosenfeld [4], Zimmerman [5], Thomson [6] and many experts in [7-12] have contributed significantly in the developments of fuzzy graphs.

Received January $12^{\text {th }}, 2021$; accepted February $15^{\text {th }}, 2021$; published March $2^{\text {nd }}, 2021$.

2010 Mathematics Subject Classification. 05C07, 05C072, $15 \mathrm{~B} 15$.

Key words and phrases. fuzzy graphs; fuzzy Zagreb indices; fuzzy Zagreb matrices; fuzzy Zagreb energies.

(C)2021 Authors retain the copyrights of their papers, and all open access articles are distributed under the terms of the Creative Commons Attribution License. 
In [13], Anjali and Mathew introduced energy of fuzzy graphs and in [14] authors introduced Laplacian energy of fuzzy graphs. In $[15,16]$, authors discussed Weiner index of fuzzy graph and found relationships between connectivity index and Wiener index of a fuzzy graph. Recently, authors in [17,18], discussed transitive blocks, Hamiltonian fuzzy graphs and their applications in fuzzy interconnection networks, human trafficking.

The paper is structured as follows: In section 2, we discuss the preliminary definitions required for the development of the content. In Section 3, we introduce the important definitions of fuzzy Zagreb indices, fuzzy Zagreb matrices and corresponding energies. Section 4 provides some bounds for the fuzzy Zagreb energies.

\section{Preliminaries}

In this section, we recall some definitions of Zagreb indices and some notions of fuzzy graphs which will play an important role in the subsequent sections of the paper. Basics of Zagreb indices can be referred in $[19,20]$. Basics of graphs and fuzzy graphs can be referred in $[4,21]$.

Definition 2.1. Let $G=(V, E)$ be a simple graph. Degree of vertex $u$ is denoted by $d_{u}$ and $e=u v \in E$ is an edge in graph $G$. First Zagreb index $M_{1}(G)$ is defined as

$$
M_{1}(G)=\sum_{u v \in E}\left[d_{u}+d_{v}\right]=\sum_{u \in V} d_{u}^{2}
$$

Second Zagreb index $M_{2}(G)$ is defined as

$$
M_{2}(G)=\sum_{u v \in E} d_{u} \cdot d_{v}
$$

and Hyper Zagreb index $H M(G)$ is defined as

$$
H M(G)=\sum_{u v \in E}\left[d_{u}+d_{v}\right]^{2}
$$

Definition 2.2. A fuzzy graph $G=(V, \sigma, \mu)$; which also can simply be denoted by $G=(\sigma, \mu)$, is a graph with vertex-membership function $\sigma: V \rightarrow[0,1]$ and edge-strength function $\mu: V \times V \rightarrow[0,1]$ such that it satisfies the relation $\mu(x, y) \leq \min \{\sigma(x), \sigma(y)\}, \forall x, y \in V$. Corresponding crisp graph is denoted by $G=\left(\sigma^{*}, \mu^{*}\right)$.

Also, we denote strength of vertex $u$ by $\mu(u)$, it represents the minimum of strengths of edges incident to the vertex $u$

$$
\mu(u)=\underset{u v_{i} \in \mu^{*}}{\wedge} \mu\left(u, v_{i}\right)
$$

\section{Fuzzy Zagreb Indices}

In this section, we introduce the definitions of fuzzy Zagreb first index, fuzzy Zagreb second index and fuzzy Zagreb hyper index along with associated fuzzy Zagreb matrices and the fuzzy Zagreb energies. These definitions are required to discuss the main results. 
Definition 3.1. The fuzzy Zagreb first index of $G=(\sigma, \mu)$ is defined as

$$
F M_{1}(G)=\sum_{u v \in \mu^{*}}[\sigma(u) \mu(u)+\sigma(v) \mu(v)]
$$

Equivalently the index can also be defined as

$$
F M_{1}(G)=\sum_{u \in \sigma^{*}}\left[\sigma(u) \mu(u) d_{u}\right]
$$

Definition 3.2. The fuzzy Zagreb second index of $G=(\sigma, \mu)$ is defined as

$$
F M_{2}(G)=\sum_{u v \in \mu^{*}}[\sigma(u) \mu(u) \cdot \sigma(v) \mu(v)]
$$

Definition 3.3. The fuzzy hyper Zagreb index of $G=(\sigma, \mu)$ is defined as

$$
F H M(G)=\sum_{u v \in \mu^{*}}[\sigma(u) \mu(u)+\sigma(v) \mu(v)]^{2}
$$

Definition 3.4. If $G=(\sigma, \mu)$ is a fuzzy graph and $\sigma^{*}=\left\{u_{1}, u_{2}, \ldots, u_{n}\right\}$ then first fuzzy Zagreb matrix is defined as $F Z^{(1)}=\left(f z^{(1)}\right)_{i, j}$, where

$$
\left(f z^{(1)}\right)_{i, j}= \begin{cases}\sigma\left(u_{i}\right) \mu\left(u_{i}\right)+\sigma\left(u_{j}\right) \mu\left(u_{j}\right) & , \text { if } i \neq j \text { and } u_{i} . u_{j} \in \mu^{*} \\ 0 & , \text { if } u_{i} . u_{j} \notin \mu^{*} \\ 0 & , \text { if } i=j\end{cases}
$$

and second fuzzy Zagreb matrix is defined as $F Z^{(2)}=\left(f z^{(2)}\right)_{i, j}$, where

$$
\left(f z^{(2)}\right)_{i, j}= \begin{cases}\sigma\left(u_{i}\right) \mu\left(u_{i}\right) \cdot \sigma\left(u_{j}\right) \mu\left(u_{j}\right) & , \text { if } i \neq j \text { and } u_{i} . u_{j} \in \mu^{*} \\ 0 & , \text { if } u_{i} . u_{j} \notin \mu^{*} \\ 0 & , \text { if } i=j\end{cases}
$$

Definition 3.5. If $G=(\sigma, \mu)$ is a fuzzy graph and $\sigma^{*}=\left\{u_{1}, u_{2}, \ldots, u_{n}\right\}$, if $F Z^{(1)}$ is the first fuzzy $Z$ agreb matrix with its eigen values $\xi_{1}^{(1)}, \xi_{2}^{(1)}, \ldots, \xi_{n}^{(1)}$ then the first fuzzy Zagreb energy is defined as

$$
F Z E^{(1)}=\sum_{i=1}^{n}\left|\xi_{i}^{(1)}\right|
$$

Definition 3.6. If $G=(\sigma, \mu)$ is a fuzzy graph and $\sigma^{*}=\left\{u_{1}, u_{2}, \ldots, u_{n}\right\}$, if $F Z^{(2)}$ is the second fuzzy Zagreb matrix with $\xi_{1}^{(2)}, \xi_{2}^{(2)}, \ldots, \xi_{n}^{(2)}$ as its eigen values then the second fuzzy Zagreb energy is defined as

$$
F Z E^{(2)}=\sum_{i=1}^{n}\left|\xi_{i}^{(2)}\right|
$$

Example 3.1. Consider the fuzzy graphs $G=(\sigma, \mu)$ as shown in fig.1 Here $\sigma^{*}=\left\{u_{1}, u_{2}, u_{3}, u_{4}, u_{5}, u_{6}\right\}$ with membership values $\sigma\left(u_{1}\right)=0.4, \sigma\left(u_{2}\right)=0.2, \sigma\left(u_{3}\right)=0.6, \sigma\left(u_{4}\right)=0.5, \sigma\left(u_{5}\right)=0.7, \sigma\left(u_{6}\right)=0.8$ and strengths of edges $\mu\left(u_{1} u_{2}\right)=0.1, \mu\left(u_{2} u_{3}\right)=0.2, \mu\left(u_{2} u_{4}\right)=0.1, \mu\left(u_{3} u_{4}\right)=0.4, \mu\left(u_{4} u_{5}\right)=0.4$, $\mu\left(u_{5} u_{6}\right)=0.5, \mu\left(u_{5} u_{1}\right)=0.3, \mu\left(u_{1} u_{6}\right)=0.3$ then we get $F M_{1}=1.68, F M_{2}=0.1107$ and $F H M=0.0468$. The first and second fuzzy Zagreb matrices are given by 


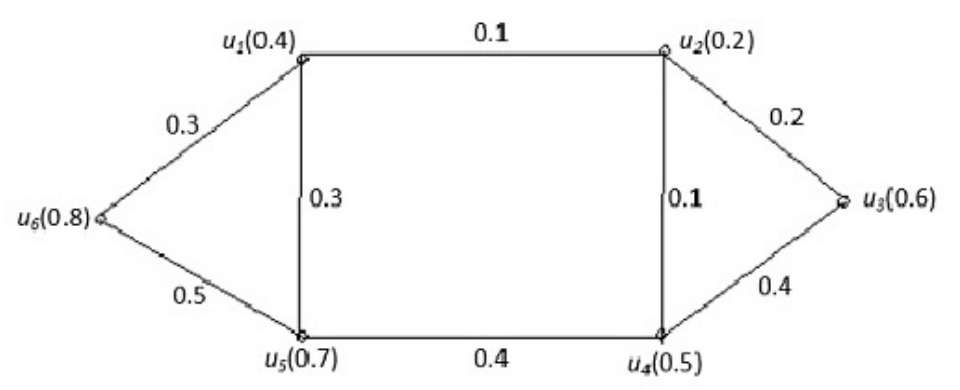

Figure 1.

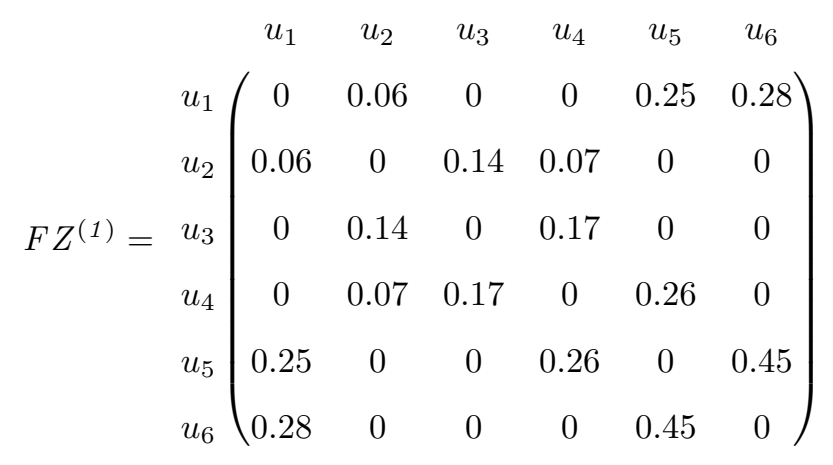

and

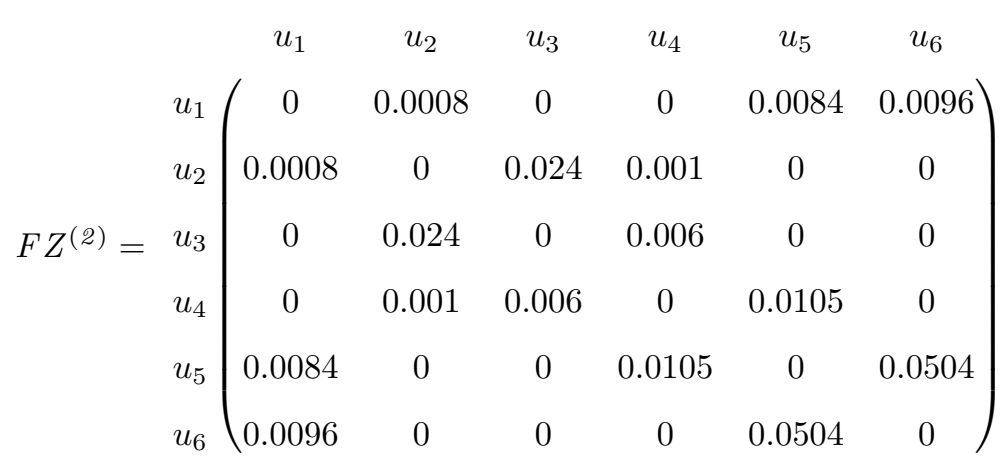

Eigen values of $F Z^{(1)}$ are given by $\xi_{1}^{(1)}=0.7067, \xi_{2}^{(1)}=-0.5276, \xi_{3}^{(1)}=-0.2595, \xi_{4}^{(1)}=0.2487$, $\xi_{5}^{(1)}=-0.1704, \xi_{6}^{(1)}=0.0021$ hence the first fuzzy Zagreb energy is $F Z E^{(1)}=1.915$.

Eigen values of $F Z^{(2)}$ are given by $\xi_{1}^{(2)}=0.0544, \xi_{2}^{(2)}=-0.0515, \xi_{3}^{(2)}=0.0249, \xi_{4}^{(2)}=-0.0245, \xi_{5}^{(2)}=$ $-0.00404, \xi_{6}^{(2)}=0.00069$ hence the second fuzzy Zagreb energy is $F Z E^{(2)}=0.16$.

\section{Main Results}

In this paper we will discuss fuzzy Zagreb first index and the corresponding first fuzzy Zagreb matrix and first fuzzy Zagreb energy. The analogous study of second fuzzy Zagreb quantities along with first and second fuzzy Eztrada Zagreb energies will be communicated in forthcoming paper. 
For simplicity of notations, fuzzy Zagreb first matrix will be denoted by $F Z$, its $(i, j)^{t h}$ element by $(f z)_{i, j}$ and the corresponding fuzzy Zagreb energy by $F Z E=\sum_{i=1}^{n}\left|\xi_{i}\right|$, where $\xi_{1} \geq \xi_{2} \geq \ldots \geq \xi_{n}$ are eigen values of $F Z$. Here $F Z$ is a real symmetric matrix and hence all its eigen values are real. For non-negative integer $k$, the $k^{t h}$ spectral moment of $F Z$ is given by $N_{k}=\sum_{i=1}^{n}\left(\xi_{i}\right)^{k}=\operatorname{tr}\left(F Z^{k}\right)$

Although elementary, the following result deserves tobe stated as:

Theorem 4.1. If $G=(V, \sigma, \mu)$ and $H=\left(W, \sigma^{\prime}, \mu^{\prime}\right)$ are fuzzy isomorphic graphs then $F M_{1}(G)=F M_{1}(H)$ and $F M_{2}(G)=F M_{2}(H)$

Proof. If $G=(V, \sigma, \mu)$ and $H=\left(W, \sigma^{\prime}, \mu^{\prime}\right)$ are fuzzy isomorphic graphs then there exists a bijective function $f: V \rightarrow W$ such that for every $u \in V$ and $u v \in \mu^{*}, \sigma(u)=\sigma^{\prime}(f(u))$ and $\mu(u v)=\mu^{\prime}(u v)$. Hence we can easily conclude that

$$
\begin{aligned}
F M_{1}(G) & =\sum_{u v \in \mu^{*}}[\sigma(u) \mu(u)+\sigma(v) \mu(v)] \\
& =\sum_{f(u) f(v) \in \mu^{\prime *}}\left[\sigma^{\prime}(f(u)) \mu^{\prime}(f(u))+\sigma^{\prime}(f(v)) \mu^{\prime}(f(v))\right] \\
& =F M_{1}(H)
\end{aligned}
$$

and also

$$
\begin{aligned}
F M_{2}(G) & =\sum_{u v \in \mu^{*}}[\sigma(u) \mu(u) \cdot \sigma(v) \mu(v)] \\
& =\sum_{f(u) f(v) \in \mu^{\prime *}}\left[\sigma^{\prime}(f(u)) \mu^{\prime}(f(u)) \cdot \sigma^{\prime}(f(v)) \mu^{\prime}(f(v))\right] \\
& =F M_{2}(H)
\end{aligned}
$$

Theorem 4.2. If $G=(V, \sigma, \mu)$ with $V=\left\{u_{1}, u_{2}, \ldots, u_{n}\right\}$ is a fuzzy graph and $F Z$ is its fuzzy Zagreb matrix then
(1) $N_{1}=\operatorname{tr}(F Z)=0$
(2) $N_{2}=\operatorname{tr}\left(F Z^{2}\right)=2 . F H M$ 
Proof. (1) By definition of $F Z$, all its diagonal elements are zero, hence trace of $F Z$ is zero.

(2) The diagonal elements of $F Z^{2}$ are given by

$$
\begin{aligned}
\left(f z^{2}\right)_{i, i} & =\sum_{j=1}^{n}(f z)_{i, j} \cdot(f z)_{j, i} \\
& =\sum_{j=1}^{n}(f z)_{i, j}^{2} \\
& =\sum_{\substack{u_{i} . u_{j} \in \mu^{*} \\
i, j \in\{1,2, \ldots, n\}}}\left[\sigma\left(u_{i}\right) \mu\left(u_{i}\right)+\sigma\left(u_{j}\right) \mu\left(u_{j}\right)\right]^{2}
\end{aligned}
$$

hence

$$
\begin{aligned}
N_{2} & =\operatorname{tr}\left(F Z^{2}\right) \\
& =\sum_{i=1}^{n} \sum_{\substack{u_{i}, u_{j} \in \mu^{*} \\
i, j \in\{1,2, \ldots, n\}}}\left[\sigma\left(u_{i}\right) \mu\left(u_{i}\right)+\sigma\left(u_{j}\right) \mu\left(u_{j}\right)\right]^{2} \\
& =2 . \sum_{\substack{u_{i}, u_{j} \in \mu^{*} \\
i, j \in\{1,2, \ldots, n\}}}\left[\sigma\left(u_{i}\right) \mu\left(u_{i}\right)+\sigma\left(u_{j}\right) \mu\left(u_{j}\right)\right]^{2} \\
& =2 . F H M
\end{aligned}
$$

Theorem 4.3. Let $G=(V, \sigma, \mu)$ be a fuzzy graph with $|V|=n$ vertices. Let $F Z$ be the corresponding fuzzy Zagreb matrix with eigen values $\xi_{1}, \xi_{2}, \ldots, \xi_{n}$ and FZE is its fuzzy Zagreb energy then

$$
\sqrt{2(F H M)+n(n-1)|\operatorname{det}(F Z)|^{\frac{2}{n}}} \leq F Z E \leq \sqrt{2 n \cdot(F H M)}
$$

\section{Proof. Upper Bound:}

Applying Cauchy-Schwartz inequality to the $n$ numbers $(1,1, \ldots, 1)$ and $\left(\xi_{1}, \xi_{2}, \ldots, \xi_{n}\right)$ we get,

$$
\begin{gathered}
\sum_{i=1}^{n}\left(1 .\left|\xi_{i}\right|\right) \leq\left(\sum_{i=1}^{n} 1\right)^{1 / 2} \cdot\left(\sum_{i=1}^{n}\left|\xi_{i}\right|^{2}\right)^{1 / 2} \\
\sum_{i=1}^{n}\left|\xi_{i}\right| \leq \sqrt{n} \cdot \sqrt{\sum_{i=1}^{n}\left|\xi_{i}\right|^{2}}
\end{gathered}
$$

Also we have,

$$
\left(\sum_{i=1}^{n}\left|\xi_{i}\right|\right)^{2}=\sum_{i=1}^{n}\left|\xi_{i}\right|^{2}+2 . \sum_{1 \leq i<j \leq n}\left|\xi_{i} \cdot \xi_{j}\right|=0
$$

hence

$$
\sum_{i=1}^{n}\left|\xi_{i}\right|^{2}=-2 . \sum_{1 \leq i<j \leq n}\left|\xi_{i} \cdot \xi_{j}\right|
$$


On comparing the coefficients of $\prod_{i=1}^{n}\left(\xi-\xi_{i}\right)=|F Z-\xi . I|$, we get

$$
\sum_{1 \leq i<j \leq n}\left|\xi_{i} \cdot \xi_{j}\right|=-\sum_{u_{i} \cdot u_{j} \in \mu^{*}}\left[\sigma\left(u_{i}\right) \mu\left(u_{i}\right)+\sigma\left(u_{j}\right) \mu\left(u_{j}\right)\right]^{2}
$$

Using (4.3), eq.(4.2) becomes

$$
\sum_{i=1}^{n}\left|\xi_{i}\right|^{2}=2 . \sum_{u_{i} . u_{j} \in \mu^{*}}\left[\sigma\left(u_{i}\right) \mu\left(u_{i}\right)+\sigma\left(u_{j}\right) \mu\left(u_{j}\right)\right]^{2}
$$

hence eq.(4.1) becomes

$$
\begin{gathered}
\sum_{i=1}^{n}\left|\xi_{i}\right| \leq \sqrt{2 n . \sum_{u_{i} . u_{j} \in \mu^{*}}\left[\sigma\left(u_{i}\right) \mu\left(u_{i}\right)+\sigma\left(u_{j}\right) \mu\left(u_{j}\right)\right]^{2}} \\
F Z E(G) \leq \sqrt{2 n \cdot(F H M)}
\end{gathered}
$$

\section{Lower Bound:}

$$
\begin{gathered}
(\operatorname{FZE}(G))^{2}=\left(\sum_{i=1}^{n}\left|\xi_{i}\right|\right)^{2} \\
=\sum_{i=1}^{n}\left|\xi_{i}\right|^{2}+2 . \sum_{1 \leq i<j \leq n}\left|\xi_{i} \cdot \xi_{j}\right| \\
(F Z E(G))^{2}=2 . \sum_{u_{i} \cdot u_{j} \in \mu^{*}}\left[\sigma\left(u_{i}\right) \mu\left(u_{i}\right)+\sigma\left(u_{j}\right) \mu\left(u_{j}\right)\right]^{2}+2 \cdot \frac{n(n-1)}{2} \cdot A M\left\{\left|\xi_{i} \cdot \xi_{j}\right|\right\}
\end{gathered}
$$

hence

$$
(F Z E(G))^{2} \geq 2 \cdot(F H M)+n(n-1) \cdot G M\left\{\left|\xi_{i} \cdot \xi_{j}\right|\right\}
$$

where

$$
\begin{aligned}
G M\left\{\left|\xi_{i} \cdot \xi_{j}\right|\right\} & =\left(\prod_{1 \leq i<j \leq n}\left|\xi_{i} \cdot \xi_{j}\right|\right)^{\frac{2}{n(n-1)}} \\
& =\left(\prod_{i=1}^{n}\left|\xi_{i}\right|^{n-1}\right)^{\frac{2}{n(n-1)}} \\
& =\left(\prod_{i=1}^{n}\left|\xi_{i}\right|\right)^{2 / n} \\
& =(\operatorname{det} F Z)^{2 / n}
\end{aligned}
$$

hence eq.(4.6) becomes

$$
(F Z E(G))^{2} \geq 2 \cdot(F H M)+n(n-1) \cdot(\operatorname{det} F Z)^{2 / n}
$$

$$
F Z E(G) \geq \sqrt{2 \cdot(F H M)+n(n-1) \cdot(\operatorname{det} F Z)^{2 / n}}
$$


Theorem 4.4. Let $G=(V, \sigma, \mu)$ be a fuzzy graph and $\sigma^{*}=\left\{u_{1}, u_{2}, \ldots, u_{n}\right\}$. If $F Z$ is the corresponding fuzzy Zagreb matrix with eigen values $\xi_{1}, \xi_{2}, \ldots, \xi_{n}$ and FZE is its fuzzy Zagreb energy then

$$
F Z E \leq \frac{2(F H M)}{n}+\sqrt{(n-1)\left\{2(F H M)-\left(\frac{2}{n}(F H M)\right)^{2}\right\}}
$$

Proof. First we recall some elementary results. If $A=\left(a_{i j}\right)_{n \times n}$ is a symmetric matrix with diagonal elements all zero then $\lambda_{\max } \geq \frac{2}{n} \sum_{1 \leq i<j \leq n} a_{i j}$, where $\lambda_{\max }$ is the maximum eigenvalue of A. Hence in case of fuzzy Zagreb matrix $F Z$, if $\xi_{1} \geq \xi_{2} \geq \cdots \geq \xi_{n}$ are its eigen values, then

$$
\left|\xi_{1}\right| \geq \frac{2}{n} \sum_{u_{i} u_{j} \in \mu^{*}}\left[\sigma\left(u_{i}\right) \mu\left(u_{i}\right)+\sigma\left(u_{j}\right) \mu\left(u_{j}\right)\right]=\frac{2}{n} F M_{1}
$$

Using eq.(4.4) of theorem 4.3,

$$
\begin{gathered}
\sum_{i=1}^{n}\left|\xi_{i}\right|^{2}=2 . \sum_{u_{i} u_{j} \in \mu^{*}}\left[\sigma\left(u_{i}\right) \mu\left(u_{i}\right)+\sigma\left(u_{j}\right) \mu\left(u_{j}\right)\right]^{2}=2(F H M) \\
\sum_{i=2}^{n}\left|\xi_{i}\right|^{2}=2(F H M)-\left|\xi_{1}\right|^{2}
\end{gathered}
$$

Using Cauchy-Schwartz inequality to the $(n-1)$ numbers $(1,1, \ldots, 1)$ and $\left(\xi_{2}, \xi_{3}, \ldots, \xi_{n}\right)$ we get

$$
\begin{gathered}
\sum_{i=2}^{n}\left(1 .\left|\xi_{i}\right|\right) \leq\left(\sum_{i=2}^{n} 1\right)^{1 / 2} \cdot\left(\sum_{i=2}^{n}\left|\xi_{i}\right|^{2}\right)^{1 / 2} \\
\sum_{i=2}^{n}\left|\xi_{i}\right| \leq \sqrt{(n-1) \cdot\left(\sum_{i=2}^{n}\left|\xi_{i}\right|^{2}\right)}
\end{gathered}
$$

Using eq.(4.10), eq.(4.11) becomes

$$
\begin{gathered}
\sum_{i=2}^{n}\left|\xi_{i}\right| \leq \sqrt{(n-1) \cdot\left\{2(F H M)-\left|\xi_{1}\right|^{2}\right\}} \\
\sum_{i=2}^{n}\left|\xi_{i}\right|+\left|\xi_{1}\right| \leq\left|\xi_{1}\right|+\sqrt{(n-1) \cdot\left\{2(F H M)-\left|\xi_{1}\right|^{2}\right\}} \\
F Z E \leq\left|\xi_{1}\right|+\sqrt{(n-1) \cdot\left\{2(F H M)-\left|\xi_{1}\right|^{2}\right\}}
\end{gathered}
$$

As the function $g(x)=x+\sqrt{(n-1) \cdot\left\{2(F H M)-x^{2}\right\}}$ is decreasing function of $x$ in the interval $\frac{2}{n}(F H M)<$ $x \leq \sqrt{2(F H M)}$ and also $\left|\xi_{1}\right| \geq \frac{2}{n} F M_{1}$, we get

$$
\sqrt{\frac{2}{n}(F H M)} \leq \frac{2}{n}(F H M) \leq \frac{2}{n} F M_{1} \leq\left|\xi_{1}\right| \leq \sqrt{2(F H M)}
$$


Hence eq.(4.12) becomes

$$
F Z E \leq \frac{2}{n}(F H M)+\sqrt{(n-1) \cdot\left\{2(F H M)-\left(\frac{2}{n}(F H M)\right)^{2}\right\}}
$$

Theorem 4.5. If $G=(V, \sigma, \mu)$ is a fuzzy graph and $\sigma^{*}=\left\{u_{1}, u_{2}, \ldots, u_{n}\right\}, F Z$ is the corresponding fuzzy Zagreb matrix with eigen values $\xi_{1} \geq \xi_{2} \geq \cdots \geq \xi_{n}>0$ and FZE is its fuzzy Zagreb energy then

$$
F Z E \geq \frac{2}{n} F M_{1}+(n-1)+\ln \left[\frac{n|\operatorname{det}(F Z)|}{2 F M_{1}}\right]
$$

Proof. Consider $G=(V, \sigma, \mu)$ is a fuzzy graph and $\xi_{1} \geq \xi_{2} \geq \ldots \geq \xi_{n}>0$ are eigen values of corresponding Fuzzy Zagreb matrix $F Z$. For simplicity, consider a function $f(x)=1-x-\ln (x), \quad x>0$. Elementary calculations shows that $f(x)$ is decreasing function in $(0,1]$ and it is increasing function for $x \geq 1$. Hence $f(x) \geq f(1)=0$ for $x>0$ gives, $x \geq 1+\ln (x)$ for $x>0$.

$$
\begin{aligned}
F Z E & =\sum_{i=1}^{n}\left|\xi_{i}\right|=\left|\xi_{1}\right|+\sum_{i=2}^{n}\left|\xi_{i}\right| \\
& \geq\left|\xi_{1}\right|+\sum_{i=2}^{n}\left[1+\ln \left|\xi_{i}\right|\right] \\
& =\left|\xi_{1}\right|+(n-1)+\ln \left[\prod_{i=2}^{n}\left|\xi_{i}\right|\right] \\
& =\left|\xi_{1}\right|+(n-1)+\ln \left[\prod_{i=1}^{n}\left|\xi_{i}\right|\right]-\ln \left|\xi_{1}\right| \\
& =\left|\xi_{1}\right|+(n-1)+\ln |\operatorname{det}(F Z)|-\ln \left|\xi_{1}\right|
\end{aligned}
$$

As the function $g(x)=x+(n-1)+\ln |\operatorname{det}(F Z)|-\ln x$ is increasing function in $1 \leq x \leq n$, hence for $\left|\xi_{1}\right| \geq \frac{2}{n} F M_{1}$,

$$
\begin{gathered}
F Z E \geq \frac{2}{n} F M_{1}+(n-1)+\ln |\operatorname{det}(F Z)|-\ln \left[\frac{2}{n} F M_{1}\right] \\
F Z E \geq \frac{2}{n} F M_{1}+(n-1)+\ln \left[\frac{n|\operatorname{det}(F Z)|}{2 F M_{1}}\right]
\end{gathered}
$$

Theorem 4.6. If $G=(V, \sigma, \mu)$ is a fuzzy graph and $\sigma^{*}=\left\{u_{1}, u_{2}, \ldots, u_{n}\right\}, F Z$ is the corresponding fuzzy Zagreb matrix with eigen values $\xi_{1} \geq \xi_{2} \geq \ldots \geq \xi_{n}>0$ and FZE is its fuzzy Zagreb energy then

$$
F Z E \leq 2(F H M)+\frac{2}{n} F M_{1}-\left(\frac{2}{n} F M_{1}\right)^{2}-\ln \left[\frac{n|\operatorname{det}(F Z)|}{2 F M_{1}}\right]
$$


Proof. Consider $G=(V, \sigma, \mu)$ is a fuzzy graph and $\xi_{1} \geq \xi_{2} \geq \ldots \geq \xi_{n}>0$ are the eigen values of $F Z$. Recall that, $2 F M_{1}=2 \sum_{u_{i} u_{j} \in \mu^{*}}\left[\sigma\left(u_{i}\right) \mu\left(u_{i}\right)+\sigma\left(u_{j}\right) \mu\left(u_{j}\right)\right] \geq n$, hence $\frac{2}{n} F M_{1} \geq 1$. For simplicity, consider a function $f(x)=x^{2}-x-\ln (x), x>0 . f(x)$ is decreasing function in $0<x \leq 1$ and it is increasing function in $x \geq 1$. Hence $f(x) \geq f(1)=0$ for $x>0$, gives $x \leq x^{2}-\ln (x)$ for $x>0$.

$$
\begin{aligned}
F Z E & =\sum_{i=1}^{n}\left|\xi_{i}\right|=\left|\xi_{1}\right|+\sum_{i=2}^{n}\left|\xi_{i}\right| \\
& \leq\left|\xi_{1}\right|+\sum_{i=2}^{n}\left[\left|\xi_{i}\right|^{2}-\ln \left|\xi_{i}\right|\right] \\
& =\left|\xi_{1}\right|+\sum_{i=1}^{n}\left|\xi_{i}\right|^{2}-\left|\xi_{1}\right|^{2}-\ln \prod_{i=1}^{n}\left|\xi_{i}\right|+\ln \left|\xi_{1}\right| \\
& =2(F H M)+\left|\xi_{1}\right|-\left|\xi_{1}\right|^{2}-\ln \left[\frac{\operatorname{det}(F Z) \mid}{\left|\xi_{1}\right|}\right]
\end{aligned}
$$

As the function $g(x)=2(F H M)+x-x^{2}-\ln [|\operatorname{det}(F Z)|]+\ln (x)$ is increasing function in $0<x \leq 1$ and it is decreasing function in $x \geq 1$, also $x \geq \frac{2}{n} F M_{1} \geq 1$ we get,

$$
F Z E \leq 2(F H M)+\frac{2}{n} F M_{1}-\left(\frac{2}{n} F M_{1}\right)^{2}-\ln \left[\frac{n|\operatorname{det}(F Z)|}{2 F M_{1}}\right]
$$

Illustration: Consider the fuzzy graph $G=(\sigma, \mu)$ as shown in fig.1. Here $F Z E=1.915, F H M=0.468$ and $F M_{1}=1.68$

$$
\begin{aligned}
F Z E & \geq \sqrt{2(F H M)+n(n-1)|\operatorname{det}(F Z)|^{\frac{2}{n}}} \\
& =\sqrt{2(0.468)+6(5)|0.00000861|^{\frac{2}{6}}} \\
& =1.2453
\end{aligned}
$$

and

$$
F Z E \leq \sqrt{2 n \cdot(F H M)}=\sqrt{2(6) \cdot(0.468)}=2.3698
$$

hence it verifies the theorem 4.3 .

$$
\begin{aligned}
F Z E & \leq \frac{2(F H M)}{n}+\sqrt{(n-1)\left\{2(F H M)-\left(\frac{2}{n}(F H M)\right)^{2}\right\}} \\
& =\frac{2(0.468)}{6}+\sqrt{(5)\left\{2(0.468)-\left(\frac{2}{6}(0.468)\right)^{2}\right\}} \\
& =2.291
\end{aligned}
$$


hence it verifies the theorem 4.4.

$$
\begin{aligned}
F Z E & \geq \frac{2}{n} F M_{1}+(n-1)+\ln \left[\frac{n|\operatorname{det}(F Z)|}{2 F M_{1}}\right] \\
& =\frac{2}{6}(1.68)+5+\ln \left[\frac{6(0.00000861)}{2(1.68)}\right] \\
& =-5.5228
\end{aligned}
$$

hence the theorem 4.5 is verified.

$$
\begin{aligned}
F Z E & \leq 2(F H M)+\frac{2}{n} F M_{1}-\left(\frac{2}{n} F M_{1}\right)^{2}-\ln \left[\frac{n|\operatorname{det}(F Z)|}{2 F M_{1}}\right] \\
& =2(0.468)+\frac{2}{6}(1.68)-\left(\frac{2}{6}(1.68)\right)^{2}-\ln \left[\frac{6(0.00000861)}{2(1.68)}\right] \\
& =12.2652
\end{aligned}
$$

hence the theorem 4.6 is verified.

\section{Applications}

In case of human trafficking, objects can be considered as vertices which are reasons for human trafficking while each link between these reasons can be considered as an edge. So each edge has strength of the routes between vertices. Concepts of indices can be applied to measure of susceptibility of certain routs which need to be eliminated with respect to human trafficking. Similarly, in case of internet routing, fuzzy Zagreb indices can be used to identify the nature of particular vertex or strength of the whole system so that it reduces the time consumption in the particular area.

\section{Conclusion}

Fuzzy Zagreb first, second and hyper indices as well as first, second fuzzy Zagreb matrices and their energies are studied. Some bounds for first fuzzy Zagreb energy are studied along with illustration. Further study on these fuzzy Zagreb indices may reveal more analogous results of these kind and will be discussed in the forthcoming papers.

Acknowledgements: The authors are highly grateful to the anonymous reviewers for their helpful comments and suggestions for improving the paper.

Conflicts of Interest: The author(s) declare that there are no conflicts of interest regarding the publication of this paper. 


\section{REFERENCES}

[1] I. Gutman, N. Trinajstić, Graph theory and molecular orbitals. total $\varphi$-electron energy of alternant hydrocarbons, Chem. Phys. Lett. 17 (4) (1972), 535-538.

[2] M. Randic, Characterization of molecular branching, J. Amer. Chem. Soc. 97 (23) (1975), 6609-6615.

[3] L. A. Zadeh, Fuzzy sets, Inform. Control. 8 (3) (1965), 338-353.

[4] A. Rosenfeld, Fuzzy graphs, in: Fuzzy sets and their applications to cognitive and decision processes, Elsevier, 1975, pp. 77-95.

[5] H.-J. Zimmermann, Fuzzy set theory and mathematical programming, in: Fuzzy Sets Theory and Applications, Springer, 1986, pp. 99-114.

[6] M. G. Thomason, Convergence of powers of a fuzzy matrix, J. Math. Anal. Appl. 57 (2) (1977), 476-480.

[7] J. N. Mordeson, P. S. Nair, Fuzzy graphs and fuzzy hypergraphs, Vol. 46, Physica Verlag, Heidelberg, 2012.

[8] M. Sunitha, A. Vijayakumar, Complement of a fuzzy graph, Indian J. Pure Appl. Math. 33 (9) (2002), 1451-1464.

[9] S. Mathew, M. Sunitha, Types of arcs in a fuzzy graph, Inform. Sci. 179 (11) (2009), 1760-1768.

[10] A. Nagoorgani, V. Chandrasekaran, A first look at fuzzy graph theory, Allied Publication Pvt. Ltd, Chennai, (2010).

[11] A. N. Gani, K. Radha, The degree of a vertex in some fuzzy graphs, Int. J. Algorithms Comput. Math 2 (2009), $107-116$.

[12] A. Nagoorgani, K. Ponnalagu, A new approach on solving intuitionistic fuzzy linear programming problem, Appl. Math. Sci. 6 (70) (2012), 3467-3474.

[13] N. Anjali, S. Mathew, Energy of a fuzzy graph, Ann. Fuzzy Math. Inform. 6 (3) (2013), 455-465.

[14] S. R. Sharbaf, F. Fayazi, Laplacian energy of a fuzzy graph. Iran. J. Math. Chem. 5 (1) (2014), 1-10

[15] M. Binu, S. Mathew, J. N. Mordeson, Wiener index of a fuzzy graph and application to illegal immigration networks, Fuzzy Sets Syst. 384 (2020), 132-147.

[16] S. R. Islam, S. Maity, M. Pal, Comment on "wiener index of a fuzzy graph and application to illegal immigration networks", Fuzzy Sets Syst. 384 (2020), 148-151.

[17] S. Mathew, N. Anjali, J. N. Mordeson, Transitive blocks and their applications in fuzzy interconnection networks, Fuzzy Sets Syst. 352 (2018), 142-160.

[18] S. Ali, S. Mathew, J. Mordeson, Hamiltonian fuzzy graphs with application to human trafficking, Inform. Sci. 550 (2021), 268-284.

[19] M. Kale, S. Minirani, On zagreb indices of graphs with a deleted edge, Ann. Pure Appl. Math. 21 (1) (2020), 1-14.

[20] B. Borovicanin, K. C. Das, B. Furtula, I. Gutman, Bounds for zagreb indices, MATCH Commun. Math. Comput. Chem 78 (1) (2017), 17-100.

[21] J. N. Mordeson, S. Mathew, Advanced Topics in Fuzzy Graph Theory, Springer, 2019. 